\title{
The role of small versus large scale magnetic topologies in global waves
}

\author{
C. Delannée $e^{1,2}$ \\ 1 Royal Observatory of Belgium, Brussels, Belgium \\ 2 Observatoire de Paris, CNRS, UPMC, Université Paris Diderot, 5 place Jules Janssen, 92190 Meudon, France \\ e-mail: ceaulanier@wanadoo.fr
}

Received 24 January 2008 / Accepted 15 July 2008

\begin{abstract}
Context. Coronal waves are large-scale structures that propagate through the lower corona over distances of hundreds of megameters. They are believed to be related to coronal mass ejections (CMEs). Attrill and collaborators suggested that the propagation of the wave front is due to consecutive reconnections in the quiet Sun of favourably orientated magnetic field lines as a magnetic flux tube expands in an active region.

Aims. I examine the validity of this mechanism describing the computed magnetic field topology underlying a coronal wave studied by Attrill and collaborators.

Methods. I perform an extrapolation of the magnetic field in and around the active region and overlay the magnetic field lines on base difference images of the coronal wave.

Results. The active region is magnetically linked to regions at a distance $300 \mathrm{Mm}$, including the northern coronal hole and the opposite hemisphere, but not to the quiet Sun surrounding the active region. The outer border of the active region is at the boundary of two different topological magnetic domains. The boundary of magnetic topological domains usually act as a barrier along which magnetic field lines can slip, but through which they cannot pass. Therefore, the quiet Sun around the active region should be barely perturbed by the motion occurring in the active region in such a pre-event magnetic field configuration.

Conclusions. In this magnetic field topology, the quiet Sun should not undergo any reconnection process due to the eruption in the active region, in contrast to the proposal of Attrill and collaborators.
\end{abstract}

Key words. Sun: activity - Sun: coronal mass ejections (CMEs) - Sun: magnetic fields

\section{Introduction}

Thompson et al. (1998) discovered large-scale waves propagating through the solar disc which were detectable in a FeXII spectral emission line at $195 \AA$. Those waves were observed by the extreme ultra-violet imaging telescope (EIT, Delaboudinière et al. 1995) onboard the solar and heliospheric observatory (SoHO). They are refered to as EIT waves. Similar global waves deteted in $\mathrm{H} \alpha$ (Moreton 1960) are called Moreton waves. Narukage et al. (2002) and Khan \& Aurass (2002) observed similar waves in soft- $X$ rays. These three types of observed waves are possibly different structures. However, Warmuth et al. (2004) showed that when a Moreton wave, a soft X-ray wave, and an EIT wave were observed during the same event, their fronts were co-spatial. Therefore, these three different waves may indeed be the same structure observable in a large range of spectral lines.

Cliver et al. (2005) noted that, during 1960-1970, Moreton waves were closely associated with flares. Based on a statistical analysis of two catalogs, Biesecker et al. (2002) found that only about $60 \%$ of EIT waves were related to a flare, in apparent contradiction to the relation between EIT waves and flares, and the relation between Moreton and EIT waves. However, the study was made by associating events mentioned in lists established by other observers, without checking in each case if the association was real on the basis of the observations themselves. In particular, an arbitrary minimum level of X-ray emission is chosen to declare that a flare occurs which Biesecker et al. (2002) did not indicate. Cliver et al. (2005) indicated that faint flares can be related to EIT waves, implying that this threshold was crucial to the reliability of the results obtained by Biesecker et al. (2005). To my knowledge, no observations have been reported of a coronal wave without any, even very faint, related flaring region. As widely proposed, I, therefore, also suggest that each coronal wave is related to a flare.

These coronal waves, observed in FeXII and soft X-ray wavelengths, were at first interpreted to be magnetosonic waves, originating in a flare and propagating freely through the solar surface (see e.g. Warmuth et al. 2007, and references therein). However, several facts are inconsistent with this model. Firstly, very few flares produce an observable wave, even among the strongest flares (Delannée \& Aulanier 1999; Cliver et al. 2005, Chen 2006). Each wave is related to a CME (Biesecker et al. 2002). Therefore the coronal waves seem to be related more to CMEs than to flares. Alternative models appear to interpret the coronal waves in terms of CME-related structures: they could be piston-driven shocks generated by the expansion of the CMEs (Warmuth et al. 2007), or brightened plasma in layers of jumps of magnetic field line connectivity (Delannée \& Aulanier 1999; Delannée et al. 2007) or consecutive reconnection between favourably orientated small-scale magnetic field lines of the quiet Sun (Attrill et al. 2007). 

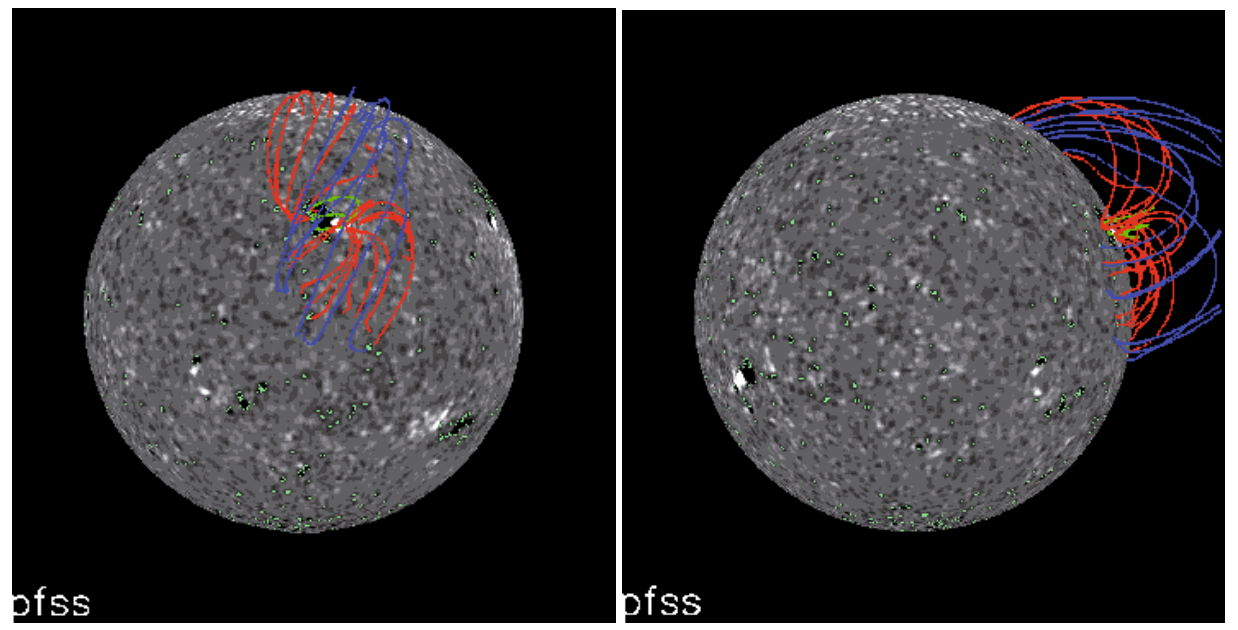

Fig. 1. Magnetic field lines extrapolated using the pfss/SSWIDL package and plotted over magnetograms obtained by MDI/SoHO. The field lines showing the different magnetic field domains are drawn in different colours. The green ones show the link between the main magnetic polarities in the active region. The red ones have one footpoint in the active region and the other one far from it. The blue ones have both footpoints close to the red magnetic field line footpoints farthest from the active region. All the magnetic field lines drawn here define the large scale magnetic field topological domains of the Sun prior to the coronal wave. The two pannels show the same magnetic field lines but in the left panel they are presented from the same point-of-view of the coronal wave and in the right panel they are presented from the side.

In this paper, I investigate in detail the final of these aforementioned models. CMEs were modeled successfully by an expanding magnetic flux tube (Aulanier et al. 2005; Lynch et al. 2005). During this expansion, Attrill et al. (2007) suggested that consecutive reconnections could occur in quiet sun loops pushed together by the expanding magnetic flux tube. The consecutive reconnections would enlarge the magnetic flux tube footprint on the solar surface. This enlargement could explain the discrepancy between the small area in which the eruption originates and the extensive area of the Sun and its corona affected by the eruption. The consecutive reconnection of small-scale magnetic field lines produces a bright structure that propagates, resembling the observed coronal waves.

In many studies, the morphologies of brightenings are explained successfully by the study of the potential magnetic field topology, even close to the flare site where it is often proposed that the magnetic field is hardly potential but instead highly stressed (Mandrini et al. 2002; Aulanier et al. 2000). Wang et al. (2002) noted that the large scale magnetic field was very close to being potential. Since Attrill et al. (2007) described their scenario, I compare its requirements with the pre-eruptive computed magnetic field topology of the Sun. I derived a map of the coronal magnetic field lines prior to the coronal wave that appeared on May 12, 1997 at 04:50 UT, and studied by Attrill et al. (2007), by using the potential field source surface package, available in SolarSoftWare, developed using the Interative Data Language package (specifically the algorithm pfss/SSWIDL) (Schrivjer \& deRosa 2003), which computes the extrapolated potential magnetic-field lines from a synoptic magnetic map obtained from Michelson Doppler Imager data (MDI/SoHO, Scherrer et al. 1995). A C-flare in AR 8038 is related to this coronal wave. Since it is related to a CME, I also discussed the possibility of dynamical triggering of the magnetic reconnection in the quiet Sun.

\section{Pre-eruptive large-scale potential magnetic field topology}

The magnetic field line extrapolation was obtained using the pfss/SSWIDL package. The synoptic map of the magnetic field at the photosphere was constructed using the MDI data. The map was updated every $6 \mathrm{~h}$ to include data close to the central meridian of the Sun. I used the synoptic map constructed for that particular day at 00:04 UT. The reconnection process should occur in the layer corresponding to the boundary between domains of different connectivity of the magnetic field lines (Démoulin et al. 1996). I was not unable to determine the precise location of this boundary using pfss/SSWIDL to extrapolate the coronal magnetic field. Therefore, the topology of the magnetic field was obtained by identifying the magnetic field lines that started close to each other and ended the most distant from each other. AR 8038 erupted on May 12, 1997 at 04:50 UT, producing a CME and a coronal wave. I was interested only in the domains surrounding AR 8038 on May 12, 1997 before the eruption.

The wave was observed using the $195 \AA$ filter of EIT. I corrected all of the images acquired during the course of the solar rotation to correspond to its position at 04:50 UT, the time of the first image obtained with EIT showing the eruption. The images of the wave were then substracted from the image acquired at 04:34 UT to derive the Derotated Base Difference Images (DBDIs, Delannée et al. 2007). I used highly contrasted DBDIs to emphasize the faintness of the wave front. The magnetic field map is also corrected for solar rotation, to match the position of the Sun at 04:50 UT, and obtain an overlay of all DBDIs and extrapolated magnetic field lines. To show the altitude extension of the magnetic field lines, I present the same extrapolated magnetic field lines viewed above the limb.

The magnetic field domains around the active region are as follows (Fig. 1). The boundary of one domain consists of small magnetic field lines linking the two main magnetic polarities of the active region (drawn in green). The boundary of the second domain consists of magnetic field lines with one footpoint in the positive main magnetic polarity of the active region and the other one very close to the equator in the southern hemisphere (drawn in red). Those magnetic field lines are transequatorial. The third domain is inside the magnetic field lines having one footpoint in the negative main magnetic polarity of the active region and the other footpoint at the border of the northern coronal hole (also drawn in red). All red magnetic field lines cross large distances on the solar surface (about $300 \mathrm{Mm}$ ), their lengths are about 

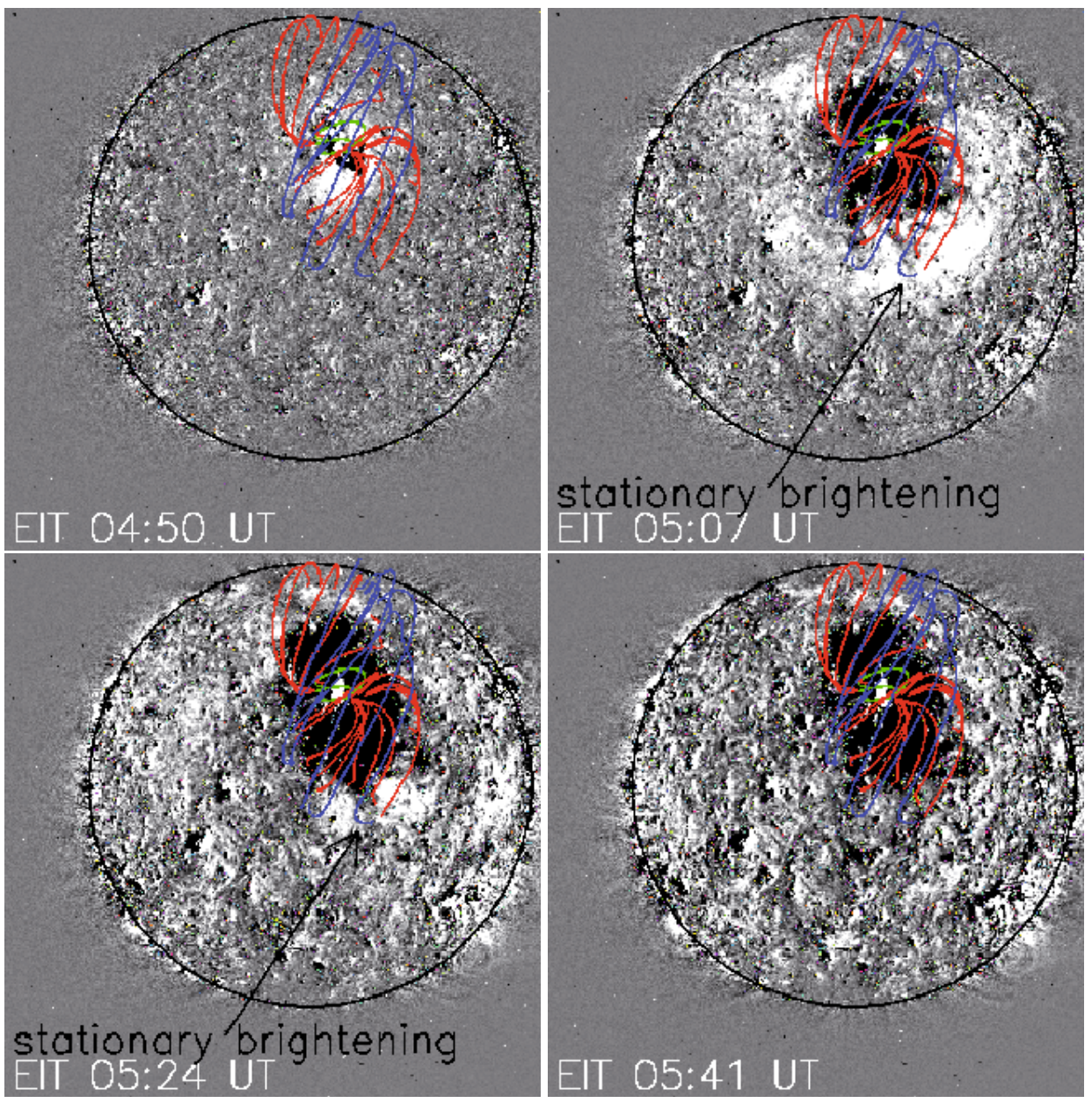

Fig. 2. DBDIs of the May 12, 1997 coronal wave overlaid with extrapolated magnetic field lines. The data is obtained using the $195 \AA$ filter of EIT/SoHO. Note the appearance of stationary brightenings at the footpoint of the red and blue magnetic field lines on the passage of the coronal wave.

$1000 \mathrm{Mm}$ and reach altitudes of about $300 \mathrm{Mm}$. Finally, the last domain is outside the magnetic field lines overlying the red ones (drawn in blue). Those magnetic field lines link the northern coronal hole and the southern hemisphere close to the equator. One of their footpoints is close to the northern footpoint of red magnetic field lines and their other footpoint is close to the southern footpoint of red magnetic field lines. This large scale topology is the same as the one simulated by Titov et al. (2008) to mimic the pre-eruptive magnetic field of the May 12, 1997 event.

Under the red magnetic field lines are plenty of magnetic field lines that connect small magnetic polarities. These small magnetic field lines form the so-called magnetic carpet of the quiet Sun. They are enclosed in small scale magnetic topological domains that belong to the second and third large scale magnetic topological domains described above. Between the green and red magnetic field lines, some small magnetic loops may exist but should be small and located in a small area. The magnetic polarities producing those loops are not observed.

I note that stationary brightenings appear during the passage of the coronal wave (Fig. 2). They remain bright for more than $17 \mathrm{~min}$ but less than $34 \mathrm{~min}$. One, described in Thompson et al. (1998), is located at the border of the coronal hole; the other one is in the southern hemisphere close to the equator (shown by the arrow in Fig. 2). Both stationary brightenings lie inside the layer separating two domains of magnetic field line connectivity, indicated by the red and blue magnetic field lines.

\section{Dynamics of the magnetic field lines in topological domains}

In the previous section, the large-scale magnetic field topology appeared to be quadrupolar. Several simulations illustrate the behavior of magnetic field lines in a similar magnetic topology as a magnetic flux tube, located under the central magnetic field domain, expands: the breakout model explains how highly sheared arcades can be ejected in quadrupolar magnetic field (Antiochos et al. 1999; MacNeice et al. 2004; Lynch et al. 2005); the ejection of a highly twisted magnetic flux tube in a quadrupolar magnetic topology was also simulated by e.g. Titov et al. (2008) and Amari et al. (2007). The quadrupolar magnetic field, used by Titov et al. (2008) as the initial condition of an eruption, corresponds as closely as possible to the extrapolated magnetic field presented in Sect. 2. While the first simulations were performed in axisymmetric and spherical coordinates, the second set were performed for three fully independent coordinates. Nevertheless, each simulations demonstrated that the expanding magnetic flux tubes are confined inside the first topological domain described in the previous section (see Fig. 1 in MacNeice et al. 2004; Fig. 10 in Titov et al. 2008; and Fig. 3 in Amari et al. 2007). 
They never crossed the boundary of their magnetic topological domain. The simulation perfomed by Titov et al. (2007) should resemble the observed ejection because the extrapolated preevent and simulated magnetic field configurations are close in their setups. One other observation combined with the extrapolation of the coronal magnetic field suggests that an ejected magnetic flux tube is confined inside the magnetic topological domains. During the ejection, the magnetic field is reconfigured, but even after the relaxation of the magnetic field configuration, close to the solar surface, the boundaries of the topological domains remain at the same locations as prior to the eruption (Gary $\&$ Moore 2004). So, the behavior of the ejected magnetic flux that cannot cross the boundary of magnetic field topological domains seems to be general.

From these simulations and observations, it is therefore difficult to imagine that, in the magnetic topology that existed before the May 12, 1997 event, the expanding magnetic flux tube, i.e. the CME, would have crossed the boundary of the first magnetic topological domain, i.e. the red magnetic-field lines, to reach the quiet sun loops located under the red magnetic field lines with which they would have reconnected to illuminate the plasma. If indeed some small magnetic field lines existed between the red and the green lines, they may have been reconnected by the same process described in Attrill et al. (2007), but these successive reconnections would stop at the footpoint of the red magnetic field lines. Therefore, the location of the bright front visible at 04:50 UT (Fig. 2) cannot be explained by successive reconnection model of these small magnetic field lines.

In each of these simulations, a reconnection process operated at a location independent of the flare: some magnetic field lines above the erupting flux tube reconnected with the tube (see the two last panels in Fig. 1 in MacNeice et al. 2004; and Fig. 3 in Amari et al. 2007). New magnetic field lines formed and glided back to the Sun. These were new magnetic field lines belonging to the second and third magnetic field topologycal domains described above. They were observed in soft X-rays during May 12, 1997 event (Attrill et al. 2006). However, large-scale ones do not form the new topology described by Attril et al. (2007). The production of these new magnetic field line were not responsible for the enlargement of the CME footprint. Blue magnetic field lines, above the ejected magnetic flux tube, pushed by the flux tube, were solely responsible for the enlargement of the CME footprint in the corona.

The expanding magnetic flux tube reconnected with above magnetic field lines. This reconnection occured when the magnetic flux tube was located at high altitude close to the blue magnetic field lines, i.e. at altitudes above $300 \mathrm{Mm}$. The particles accelerated at the reconnection site followed the new red magnetic field lines and heated the chromosphere at their footpoint, i.e. at the boundary of the coronal hole and close to the equator, illuminating the plasma in this aera. This process resembles the one described by Attrill et al. (2007), although it cannot explain the illumination of the plasma that appeared between the active region and the equator or the boundary of the coronal hole as in the image at 4:50 UT in Fig. 2.

Let us imagine some scenarios explaining how the expansion of a magnetic flux tube located under the green magnetic field lines would produce some subsequent magnetic reconnections in the magnetic carpet. In the simulations cited here, the expanding flux tube inflated not only upwards but also sideways, compressing the magnetic field lines that are at its sides. This sideways compression was fully described by Delannée et al. (2008). If the sideways magnetic field compression was sufficiently strong to compress the magnetic carpet, a reconnection among magnetic field lines in the magnetic carpet or between largescale magnetic field lines and the magnetic field lines of the magnetic carpet could occur. However, simulations of the ejection of a magnetic flux tube described above, clearly show that this compression was faint. This faint side compression was consistent with the self similar expansion of CMEs (Maričić et al. 2004). The side compression could be seen as slightly brightened plasma, as explained by Delannée et al. (2008), but in all the simulations it was hardly able to push, in a significant way, the magnetic field lines in the magnetic domain at the sides of the erupting magnetic flux tube. I note that if some conditions do produce the reconnections in the magnetic carpet as described above they must be found and studied. In all cases, the magnetic carpet appears to have a complex topology, with entangled magnetic field loops, and not a straightforward pattern. It is therefore difficult to explain how successive reconnections in a random distribution of magnetic-field lines could produce the apparent progression of the wave front.

\section{Results and conclusions}

The extrapolations of magnetic field lines prior to eruptions have been successfully used in previous studies to explain the appearance of structures during eruptions. I have performed potential magnetic field extrapolations because, even if the magnetic carpet is stressed, leading to complex topologies, the large-scale magnetic field should remain primarily potential. A comparison of observed transequatorial loop morphologies with the extrapolated potential magnetic field lines supports this argument: the morphologies with those both are in good agreement. Those large-scale magnetic field lines are likely to be present at the beginning of the eruption. The magnetic field topology about AR 8038 before the eruption is multipolar as in the cartoon presented Attril et al. (2007). Despite this apparent resemblence, AR 8038 is not magnetically connected to the surrounding quiet sun but instead to regions $300 \mathrm{Mm}$ away. The large-scale magnetic field lines located at the border of the active region separate different topological magnetic domains. Those boundaries have been shown to act like walls when magnetic field lines inside a topological magnetic domain are moving.

When the flux tube erupted, it pushed the magnetic field lines at its sides. The motion in the large-scale magnetic field lines was then possibly able to generate motion in the magnetic carpet. However, existing simulations of erupting magnetic flux tubes indicate that this sideways motion is insufficient to compress the side magnetic field lines to enable them to reach very low altitudes. If it were possible for efficient side compression to produce reconnection in the quiet sun, it should be illuminated. However, the propagation of the brightening in this context remain elusive because the magnetic field lines of the quiet Sun are entangled. All of these features, i.e. the magnetic field topology around the active region, the possible motion of the expanding magnetic flux tube and the absence of a regular patern in the magnetic carpet, indicate that the mechanism of successive magnetic reconnection in the quiet Sun proposed by Attrill et al. (2007) to explain the EIT wave propagation may not provide an adequate description and explanation of the observations considered in this study.

\section{References}

Amari, T., Aly, J. J., Mikic, Z., \& Linker, J. 2007, ApJ, 671, L189 Antiochos, S. K., DeVore, C. R., \& Klimchuk, J. A. 1999, ApJ, 510, 485 
Attrill, G., Nakwacki, M. S., Harra, L. K., et al. 2006, Sol. Phys., 238, 117

Attrill, G., Harra, L., van Driel-Gesztelyi, L., \& Démoulin, P. 2007, ApJ, 656, L101

Aulanier, G., DeLuca, E. E., Antiochos, S. K., McMullen, R. A., \& Golub, L. 2000, ApJ, 540, 1126

Biesecker, D. A., Myers, D. C., Thompson, B. J., Hammer, D. M., \& Vourlidas, A. 2002, ApJ, 569, 1009

Chen, P. F. 2006, ApJ, 641, L153

Cliver, E. W., Laurenza, M., Storini, M., \& Thompson, B. J., 2005, ApJ, 631, 604

Delaboudinière, J.-P., Artzner, G. E., Brunaud, J., et al. 1995, Sol. Phys., 162, 291

Delannée, C., \& Aulanier, G. 1999, Sol. Phys., 190, 107

Delannée, C., Hochedez, J.-F., \& Aulanier, G. 2007, A\&A, 465, 603

Delannée, C., Törörk, T., Aulanier, G., \& Hochedez, J.-F. 2008, Sol. Phys., 247, 123

Démoulin, P., Hénox, J. C., Priest, E. R., \& Mandrini, C. H. 1996, A\&A, 308, 643

Gary, G. A., \& Moore, R. L. 2004, ApJ, 611, 545
Khan, J. I., \& Aurass, H. 2002, A\&A, 383, 1018

Lynch, B. J., Antiochos, S. K., MacNeice, P. J., Zurbuchen, T. H., \& Fisk, L. A. 2004, ApJ, 617, 599

MacNeice, P., Antiochos, S. K., Phillips, A., et al. 2004, ApJ, 614, 1028

Mandrini, C. H., Démoulin, P., Schmieder, B., Deng, Y. Y., \& Rudawy, P. 2002, A\&A, 391, 317

Maričić, D., Vršnak, B., Stanger, A. L., \& Veronig, A. 2004, Sol. Phys., 225, 337 Moreton, G. E. 1960, ApJ, 65, 494

Narukage, N., Hudson, H. S., Morimoto, T., et al. 2002, ApJ, 572, L109

Scherrer, P. H., Bogart, R. S., Bush, R. I., et al. 1995, Sol. Phys., 162, 129

Schrivjer, C., \& deRosa, M. 2003, Sol. Phys., 212, 165

Titov, V. S., Mikic, Z., Linker, J. A., \& Lionello, R. 2008, ApJ, 675, 1614

Thompson, B. J., Plunkett, S. P., Gurman, J. B., et al. 1998, GRL, 25, 14, 2461

Wang, T., Yan, Y., Wang, J., \& Shibata, K. 2002, ApJ, 572, 580

Warmuth, A. 2007, in The High Energy Solar Corona: Waves, Eruptions, Particles, ed. L. Klein, A. MacKinnon (Springer Berlin: Verlag), Lecture Notes in Physics, 725, 107

Warmuth, A., Vršnak, B., Magdalenić, J., Hanslmeier, A., \& Otruba, W. 2004, A\&A, 418, 1101 